\title{
Understanding How Biodiversity Is Distributed in Space and Time
}

\author{
Hélène Morlon \\ Institut de Biologie de l'Ecole Normale Supérieure, UMR 8197 CNRS, 46 rue d'Ulm, \\ 75005 Paris, France \\ morlon@biologie.ens.fr \\ http://www.biologie.ens.fr/phyloeco/index.html
}

\begin{abstract}
Attention to biodiversity issues has been growing in the recent years. Despite the urgency of the problem, the development of a general theory of biodiversity is still underway. How can we develop such a theory? Two main approaches have dominated the field: the first approach has emphasized ecological controls of biodiversity, and has sought to explain static biodiversity patterns, often referred to as macroecological patterns; the second approach has emphasized historical controls of biodiversity, and has sought to explain more dynamic, evolutionary patterns of biodiversity, often referred to as macroevolutionary patterns. I will quickly summarize these approaches, focusing on the analytical and computational methods that have been used. Then, I will discuss how we can hope to integrate these two approaches to obtain a theory of biodiversity that better accounts for both historical and ecological factors.
\end{abstract}

Keywords: biodiversity theory, macroecology, macroevolution.

Ecology has started with a Linnaean period of discovering, naming, and counting species on Earth. From this descriptive effort, universal patterns have emerged, such as the way species richness increases with area, the way species abundances are distributed within communities, the way community composition changes from place to place, or the way species richness varies along latitudinal, altitudinal or environmental gradients [1]. Understanding these universal macroecological patterns has been the focus of early theoretical work, which has therefore aimed to predict static diversity patterns. To illustrate the type of models and the analytical and computational methods that ecologists resort to predict these macroecological patterns, I will focus on one of the theories that has received most attention from theoreticians: the neutral theory of biodiversity (Fig. 1, [2]).

At the same time that ecologists documented biodiversity patterns for existing species across the surface of the Earth, paleobiologists documented temporal patterns in the fossil record, such as how the number of species, or genera, varied through time. Understanding these temporal dynamics has spurred the development of another type of models, so-called birth-death models of cladogenesis. Later, these macroevolutionary models have been used far beyond the field of paleobiology, becoming central to most macroevolutionary studies [4]. In particular, they have successfully been applied to analyse long-term diversity dynamics

F. Fages and C. Piazza (Eds.): FMMB 2014, LNBI 8738, pp. 36-39, 2014.

(C) Springer International Publishing Switzerland 2014 


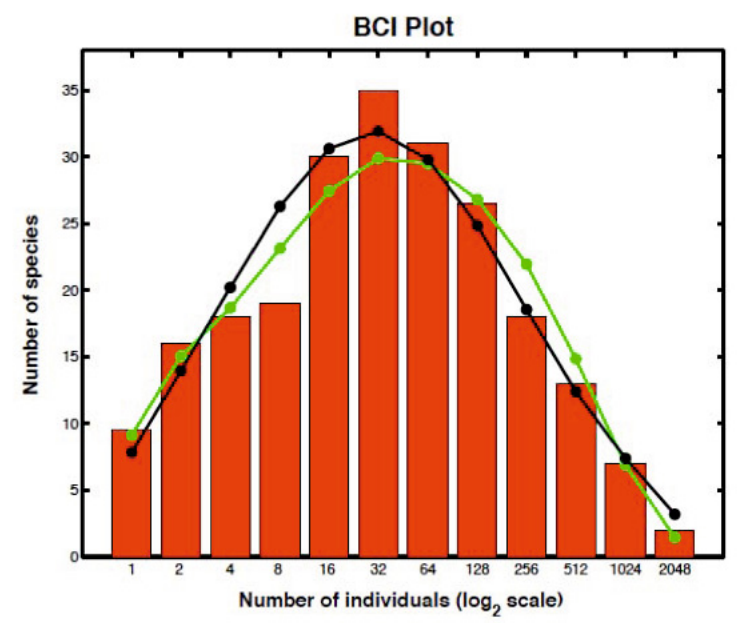

Fig. 1. An example of macroecological data used to test biodiversity theories. The red bars represent observed numbers of tree species binned into abundance categories in a 50-hectare forest plot in Barro Colorado Island. This macroecological pattern is well known as the species abundance distribution. The black and green curves represent the best fit of two different models. The neutral theory of biodiversity can be fitted to such data by maximum likelihood. From [3].

using molecular phylogenetic data. These models are now the basis of most phylogenetic methods used to understand why some groups of organisms are more species rich than others, why some geographic regions are more species rich than others, and what controls temporal variations in species richness [5]. I will detail the analytical and computational methods used to fit these macroevolutionary models to empirical data, focusing on work from my research group (Fig. 2, $[6-10])$.

While macroecological and macroevolutionary models have been developed separately, with little connections between the two fields, there is a need for an integration of these two types of models. Indeed, in their current form, macroecological models are not especially useful for understanding why species richness varies across taxonomic groups and geographic regions, while macroevolutionary models are not especially useful for understanding the distribution and abundance of species among communities. I will present news results in this direction, and discuss ways forward for a better integration of macroecological and macroevolutionary models. 
A
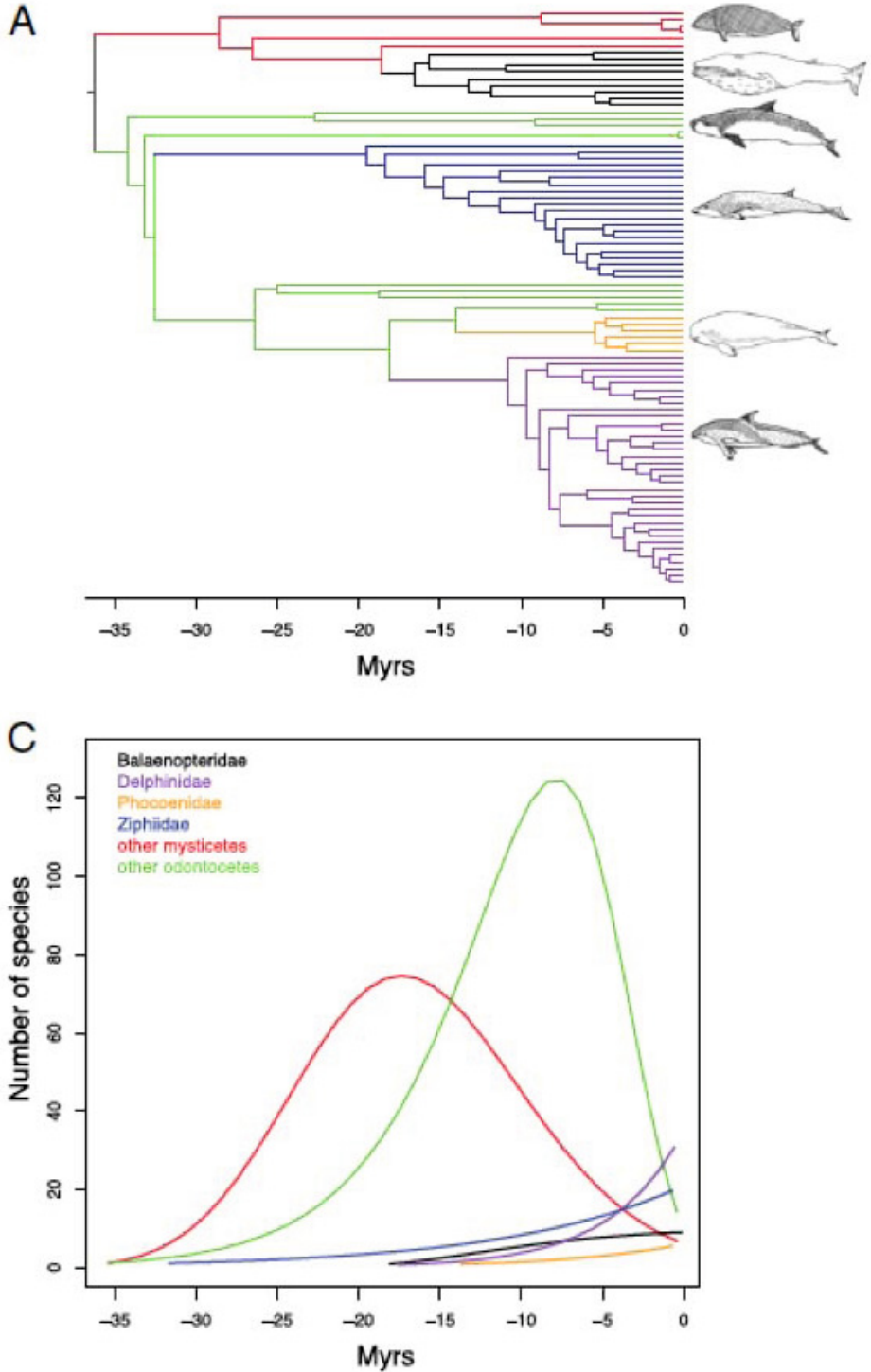

Fig. 2. An example of macroevolutionary data used to test biodiversity theories. The upper panel represents the phylogeny (dated evolutionary tree) of cetaceans (whales, dolphins and porpoises). The lower panel represents the dynamics of diversity (species richness through time) that can be inferred from this phylogenetic data, using birthdeath models of cladogenesis. From [7]. 


\section{References}

1. Rosenzweig, M.L.: Species diversity in space and time. Cambridge University Press (1995)

2. Hubbell, S.P.: The unified neutral theory of biodiversity and biogeography. Princeton University Press (2001)

3. Volkov, I., Banavar, J.R., Hubbell, S.P., Maritan, A.: Neutral theory and relative species abundance in ecology. Nature 424, 1035-1037 (2003)

4. Nee, S.: Birth-death models in macroevolution. Ann. Rev. Ecol. Evol. Syst., 1-17 (2006)

5. Morlon, H.: Phylogenetic approaches for studying diversification. Ecol. Lett., 508$525(2014)$

6. Morlon, H., Potts, M.D., Plotkin, J.B.: Inferring the dynamics of diversification: a coalescent approach. PLoS B., e1000493 (2010)

7. Morlon, H., Parsons, T.L., Plotkin, J.B.: Reconciling molecular phylogenies with the fossil record. Proc. Nat. Acad. Sci. USA, 16327-16332 (2011)

8. Condamine, F.L., Rolland, J., Morlon, H.: Macroevolutionary perspectives to environmental change. Ecol. Lett., 72-85 (2013)

9. Rolland, J., Condamine, F.L., Jiguet, F., Morlon, H.: Faster speciation and reduced extinction in the tropics contribute to the mammalian latitudinal diversity gradient. PLoS Biology, e1001775 (2014a)

10. Rolland, J., Jiguet, F., Jonsson, K.A., Condamine, F.L., Morlon, H.: Settling down of seasonal migrants promotes bird diversification. Proc. Royal Soc. B, 20140473 (2014b) 